\title{
$\mathrm{Sr}\left(\mathrm{Fe}_{1-\mathrm{x}} \mathrm{Ti}_{\mathbf{x}}\right) \mathbf{O}_{y}$ 系列材料的 穆斯堡尔谱学研究
}

\author{
郑裕芳 余正方庄严林行政 朱东燕 \\ (中山大学物理系,广州) (广州通信研究所)
}

\section{一、引 立}

钉钛矿型 $\left(\mathrm{ABO}_{3}\right)$ 化合物由于它们具有十分有用的介电的和磁的性质而受到人们广泛 的注意.

关于 $\mathrm{SrFeO}_{y}$ 的穆斯堡尔谱曾有报道 ${ }^{[1,2]}$, 但对 $\mathrm{Sr}\left(\mathrm{Fe}_{1-x} \mathrm{Ti}_{x}\right) \mathrm{O}_{y}$ 系列材料的穆斯堡尔谱学 研究的报道迄今尚未见到.

本工作研究了属于钻针矿型结构的 $\mathrm{Sr}\left(\mathrm{Fe}_{1-x} \mathrm{Ti}_{x}\right) \mathrm{O}_{y}$ 系列 $(x=0.0,0.1,0.2,0.3,0.4,0.5$, $0.6,0.7,0.8,0.9,0.95,0.99 ; y \lesssim 3)$ 材料的室温和 $78 \mathrm{~K}$ 时的穆斯堡尔谱. 着重研究材料中

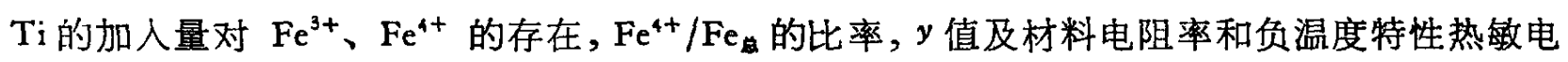
阻材料常数 $B$ 的影响. 发现当 $0 \leqslant x \leqslant 0.6$ 时穆斯堡尔谱上出现三价铁引起的组分: $\mathrm{Fe}^{3+}$ (I) 及 $\mathrm{Fe}^{3+}(\mathrm{II})$; 当 $x \geqslant 0.7$ 时, $\mathrm{Fe}^{3+}$ (II) 组分消失. 在 $x=0.9$ 至 0.99 范围内, 材料中仍 有相当大比例的 $\mathrm{Fe}^{4+}$ 存在,这与 Clevenger ${ }^{[3]}$ 的化学分析结果不一致.

\section{二、实 验}

取 $\mathrm{CP}$ 级 $\mathrm{SrCO}_{3}$ (纯度为 98.16\%), $\mathrm{CP}$ 级 $\mathrm{Fe}_{2} \mathrm{O}_{3}$ (纯度为 $99.86 \%$ ) 和 $\mathrm{CP}$ 级 $\mathrm{TiO}_{2}$ (纯 度为 $99.97 \%)$, 按 $\mathrm{Sr}\left(\mathrm{Fe}_{1-x} \mathrm{Ti}_{x}\right) \mathrm{O}_{3}(x=0.0,0.1,0.2,0.3,0.4,0.5,0.6,0.7,0.8,0.9,0.95$, 0.99) 配方, 其中当 $x=0.99$ 时采用增丰 ${ }^{57} \mathrm{Fe}_{2} \mathrm{O}_{3}$ 粉末，采用一般陶瓷工艺,在大气条件下于 $1080^{\circ} \mathrm{C}$ 预烧 $2 \mathrm{~h}$, 终烧温度 $1250 \sim 1400^{\circ} \mathrm{C}$.

电阻率测量采用电阻电桥和数字繁用表, 低阻采用四端电位法测量, 由台式自动平衡记录 仪记录.

将样品在玛瑙钵中研磨至 180 目, 用分析天平称重 $220 \mathrm{mg}$, 制成 $\phi 16 \mathrm{~mm}$ 的圆形样品. 采用英国 Oxford 公司的 MS-500 型穆斯堡尔谱仪测量室温和 78K 时的穆斯堡尔谱. 所用 放射源为强度约 $20 \mathrm{mCi}$ 的以 $\mathrm{Rh}$ 为基体的 ${ }^{57} \mathrm{Co}$ 穆斯堡尔源, 探测器采用 $\mathrm{RS}-\mathrm{P} 3-1603$ 型正 比计数器, 低温恒温器为 DN1726 型. 使用 MVC 450 激光测速装置标定速度. 在计算机 上使用 MOSFUN 程序对穆斯堡尔谱线进行拟合.

\section{三、结果和讨论}

图 1 为钛加入量等于 $0(x=0.0)$ 、终烧温度为 $1350^{\circ} \mathrm{C}$ 的样品在室温和 $78 \mathrm{~K}$ 温度时的穆

本文 1986 年 6 月 30 日收到. 

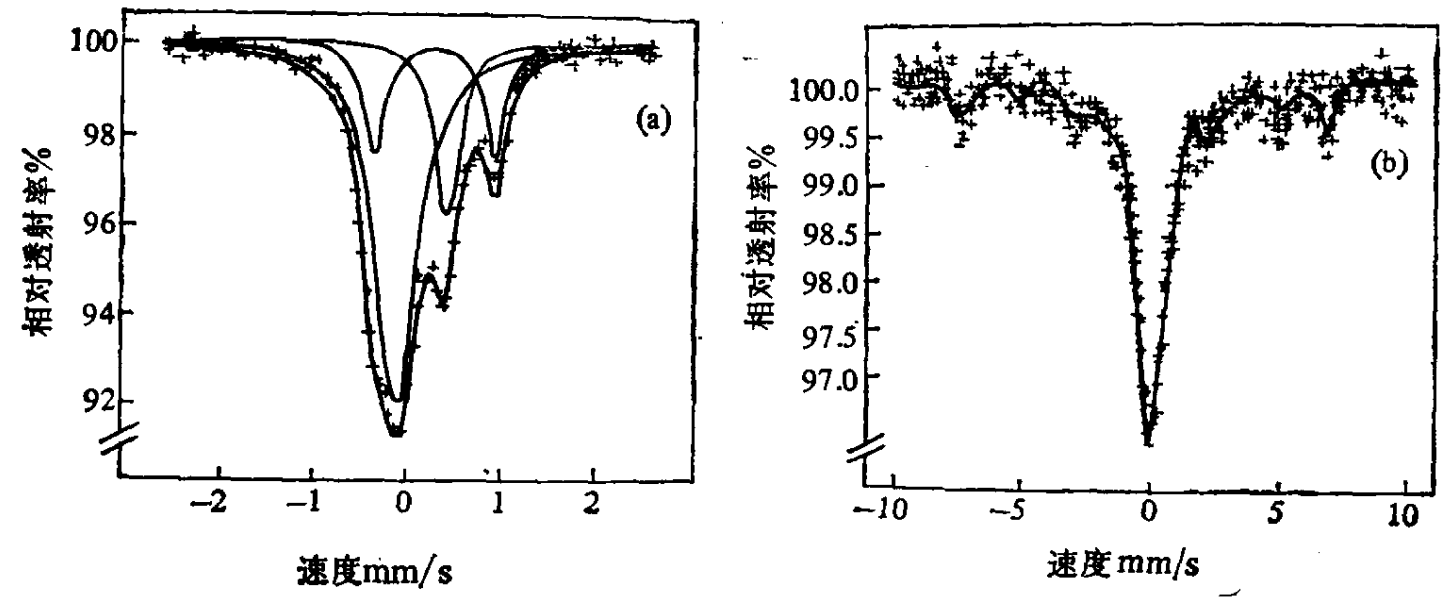

图 $1 \mathrm{SrFeO}_{2.80}$ 之穆斯堡尔谱

(a) 室温时；（b） $78 \mathrm{~K}$ 温度时

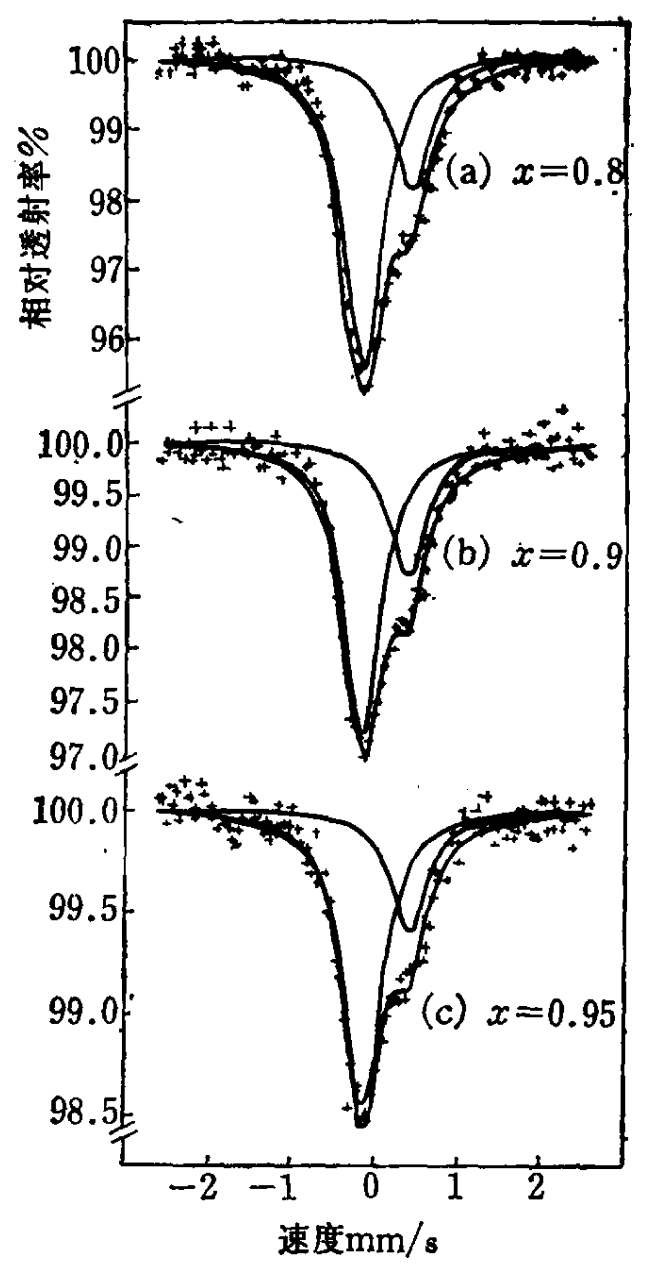

图 2 当 $x=0.8,0.9,0.95$ 时的穆斯堡尔谱

(a) $x=0.8$, 结构式为 $\mathrm{Sr}\left(\mathrm{Fe}_{0.2} \mathrm{Ti}_{0.8}\right) \mathrm{O}_{2.91} ;$ (b) $x=0.9$, 结构式为 $\mathrm{Sr}\left(\mathrm{Fe}_{0.1} \mathrm{Ti}_{0.9}\right) \mathrm{O}_{2 ., 2}$;

(c) $x=0.95$, 结构式为 $\operatorname{Sr}\left(\mathrm{Fe}_{0.09} \mathrm{Ti}_{0.93}\right) \mathrm{O}_{2.9}$

斯堡尔谱. 图 1(a) 示出三种亚谱,其成分为: 单峰 $\mathrm{Fe}^{4+}$ (同质异能移位 $1 . S .=-0.11 \mathrm{~mm} / \mathrm{s}$, 此值是相对于 $\mathrm{Rh}$ 而言, 误差为 $\pm 0.01 \mathrm{~mm} / \mathrm{s}$, 下同); 单峰 $\mathrm{Fe}^{3+}(\mathrm{I})(\mathrm{I} . \mathrm{S} .=0.39 \mathrm{~mm} / \mathrm{s})$; 
双峰 $\mathrm{Fe}^{3+}$ (II) (I.S. $=0.26 \mathrm{~mm} / \mathrm{s}$ ，四极分裂 $\mathrm{Q} . \mathrm{S} .=1.28 \mathrm{~mm} / \mathrm{s}$ ). 实验所得的这些 $\mathrm{Fe}^{4+}$ 和 $\mathrm{Fe}^{3+}$ 成分的同质异能移位值都在文献 [4] 所报道的铁化合物中 $\mathrm{Fe}^{4+}$ 和 $\mathrm{Fe}^{3+}$ 的同质异能移 位值范围内. $\mathrm{Fe}^{4+}$ 成分占材料中 $\mathrm{Fe}$ 总量百分比为 $60 \%$, 可算得样品的非理想配比结构式为 $\mathrm{SrFeO}_{2.80}$. 图 1(b) 为 $\mathrm{SrFeO}_{2.80}$ 在 $78 \mathrm{~K}$ 时的穆斯堡尔谱, 其中磁分裂部分的超精细场 $H_{i}=$ $435 \pm 2 \mathrm{KOe}, \mathrm{I} . \mathrm{S} .=0.34 \mathrm{~mm} / \mathrm{s}, \mathrm{Q} . \mathrm{S} .=-1.28 \mathrm{~mm} / \mathrm{s}$, 这是来自 $\mathrm{Fe}^{3+}$ 的贡献; 单峰 $\mathrm{Fe}^{4+}$ 成分的 I.S. $=-0.10 \mathrm{~mm} / \mathrm{s}$, 与 $\mathrm{Fe}^{4+}$ 相关的磁分裂部分因强度太微弱, 从实验谱中难于分 辨出来。

在 $x=0.0$ 至 0.6 之间, 实验所得的 $\mathrm{Fe}^{4+}$ 成分占材料中 $\mathrm{Fe}$ 总量百分比为 $60 \%$ 左右, 也 即 $\mathrm{Ti}$ 的加人量基本上不影响 $\mathrm{Fe}^{4+} / \mathrm{Fe}_{\mathrm{g}}$ 比率, 这点与 Clevenger 的化学分析结果相一致 ${ }^{[3]}$. 但 我们得出的 $\mathrm{Fe}^{3+}$ 中, 则皆含具有四极分裂的 $\mathrm{Fe}^{3+}$ (II) 成分, 这是 Clevenger 应用化学分析 方法所无法区分开的.

众所周知, 理想配比的 $\mathrm{SrFeO}_{3}$ 由氧八面体层组成, 因此在 $\mathrm{SrFeO}_{3}$ 中之 $\mathrm{Fe}^{4+}$ 具有均匀 的八面体位置. 在大气条件下烧制而成的材料中,分子结构式是非理想配比的. 其中 $\mathrm{Fe}^{3+}$ 离 子在氧八面体和四面体之间分布,此时氧的空位浓度较大, 从而导致晶体点阵之扩展, 产生较 大的四角畸变,这就是在这些材料的穆斯堡尔谱中出现 $\mathrm{Fe}^{3+}$ 四极分裂之原因.

由于在 $\mathrm{Sr}\left(\mathrm{Fe}_{1-x} \mathrm{Ti}_{x}\right) \mathrm{O}_{y}$ 系列材料中, $\mathrm{Ti}$ 总是以 $\mathrm{Ti}^{4+}$ 形式出现 ${ }^{[3]}$, 故随着 $\mathrm{Ti}$ 的加人量 $x$ 增大, 空之位浓度相应地减少, 共价键效应增强, 导致 $\mathrm{Fe}^{3+}$ 之四极分裂成分减少. 实验表 明, 当 $x \geqslant 0.7$ 时,材料之 $\mathrm{Fe}^{3+}(\mathrm{II})$ 不再存在.

图 2 为 $x=0.8,0.9,0.95$ 时材料的室温穆斯堡尔谱, 它们都由两种亚谱所组成: 左边 的单峰对应于 $\mathrm{Fe}^{4+}$ 成分, 其同质异能移位 I. S. 值分别为 $-0.16,-0.17,-0.15 \mathrm{~mm} / \mathrm{s}$; 右 边的单峰对应于 $\mathrm{Fe}^{3+}$ 成分, 其同质异能移位值分别为 $0.42,0.41$ 和 $0.42 \mathrm{~mm} / \mathrm{s}$. 显然, 这些

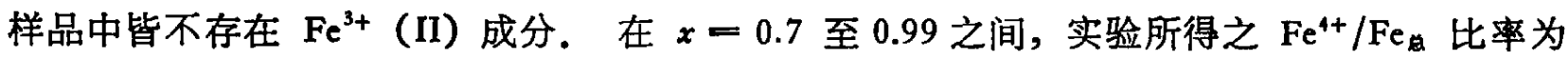
$70 \%$ 左右. 这与 Clevenger 应用化学分析所得的结果(当 $x \geqslant 0.9$ 时, $\mathrm{Fe}^{4+}$ 含量 为 0 ) 不一 致. 我们认为,他的结果是受化学分析灵敏度所限制的.

图 3 为 $\mathrm{Sr}\left(\mathrm{Fe}_{1-x} \mathrm{Ti}_{x}\right) \mathrm{O}_{y}$ 系列中的 $\mathrm{Fe}_{\text {品 }} \mathrm{Fe}^{4+}$ 的含量 (wt \% ) 和 $y$ 值随 $\mathrm{Ti}$ 含量 $x$ 的变化 关系图. 当 $x=0$ 时, $\mathrm{Fe}^{4+} / \mathrm{Fe}_{\text {㒸 }}=60 \%, y=2.80$, 较文献 $[1,3]$ 所报道的 $\mathrm{Fe}^{4+} / \mathrm{Fe}_{\mathrm{a}}=$

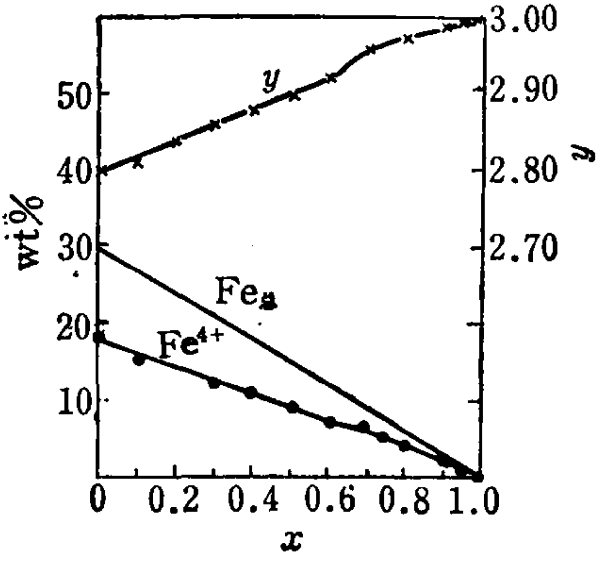

图 $3 \mathrm{Fe}_{\mathrm{H}} 、 \mathrm{Fe}^{4+}$ 的含量 $(\mathrm{wt} \%)$ 和 $y$ 值与 $x$ 的关系

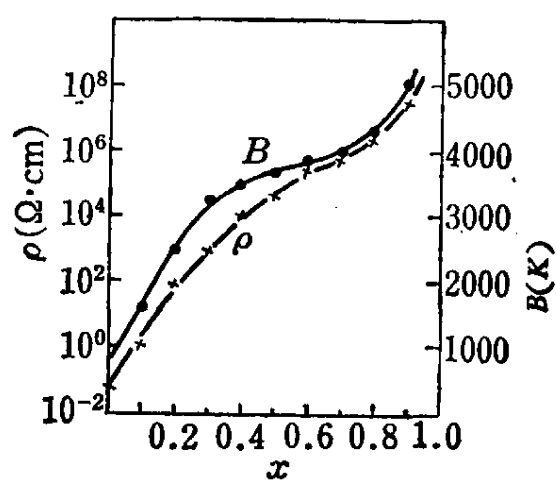

图 4 室温情况下材料电阻率 $\rho$ 和负温度特 性热敏材料常数 $B$ 值与 $\mathrm{Ti}$ 含表 $x$ 之关系 
$68 \%, y-2.86$ 值为低, 这是由于样品烧制条件不同,他们是在 1 个大气压氧气中进行的, 而 我们是在 1 个大气压空气中进行的, 故他们烧制出来的样品的氧的空位浓度较低, 使 $\mathrm{Fe}^{4+}$ 在 总 $\mathrm{Fe}$ 中的比率增大, 所以这一结果是合理的. 从图中可见, 当 $x=0.6,0.7$ 时, 由于 $\mathrm{Fe}^{4+} / \mathrm{Fe}_{\mathrm{e}}$ 比率有较大变化 (从 $60 \%$ 改变为 $70 \%$ ), 所以相应地使 $y$ 值在该处发生较大的变化.

图 4 表示室温下不同 $\mathrm{Ti}$ 含量样品之电阻率 $\rho$ 和负温度特性热敏材料常数 $B$ 值之变化曲 线. 可见,当 $x$ 增大时, $\rho$ 和 $B$ 值也皆增大. 由于 $\mathrm{Fe}^{4+}-\mathrm{Fe}^{3+}$ 的存在导致高电导率 ${ }^{[3]}$, 随着 $x$ 值 增大, 即 $\mathrm{Ti}$ 的加人量增多, $\mathrm{Fe}^{4+}$ 和 $\mathrm{Fe}^{3+}$ 的绝对含量减少, 从而 $\rho$ 值也就相应地增大. 关于 $B$ 值随 $\mathrm{Ti}$ 含量 $x$ 而变化的机制, 尚需进一步的研究.

$\mathrm{Sr}\left(\mathrm{Fe}_{1-x} \mathrm{Ti}_{x}\right) \mathrm{O}_{y}$ 系列原材料价格低廉,可采用一般陶瓷工艺在大气条件下烧成, 电阻率 $\rho$ 和材料常数 $B$ 值较易调整, 是一种有希望的 NTC (负温度系数) 材料.

\section{考文献}

[1]. Shirane, G. and Cox, D. E., Phys. Rev, 125(1962), 1158.

[2] Gallagher, P. K., MacChesney, J. B. and Buchanan, D. N. E., J. Chem. Phys, 11(1964), 2429.

[3] Clevenger, Jr. T. R., J. Am. Ceram. Soc, 46(1963), 207.

[4] Fluck, E., Kerler, W. and Neuwirth, W., Angew. Chem. Intern. Ed. Engl., 2(1963), 277.

[5] Waugh, J. S., Technical Report No. 152, Laboratory for Insulation Research, Massachusetts Institute of Technology, August 1960. 